\title{
Análisis del estado emocional de una unidad del ejército español en zona de operaciones
}

\author{
Pérez Hidalgo A. M. ${ }^{1}$, Rodríguez González JM. ${ }^{2}$
}

Sanid. mil. 2011; 67 (2): 71-77; ISSN: 1887-8571

\begin{abstract}
RESUMEN:
Introducción: El personal Militar por la naturaleza del trabajo que realiza y los lugares en los que los lleva a cabo, se convierte en una población vulnerable a padecer cambios emocionales como consecuencia del afrontamiento de dichas situaciones. Material y métodos: Se estudia un contingente de 168 miembros (edad media de 27 años, 12\% mujeres y 88\% hombres) del ejército español desplazado durante 4 meses en el año 2005 a la antigua Yugoslavia, con el objetivo de analizar las modificaciones generadas en los estados emocionales negativos de los citados individuos. La recogida de datos tuvo lugar en dos momentos diferenciados: durante la fase de concentración previa al despliegue y en una secuencia temporal posterior; al regreso a territorio nacional. En ambas ocasiones se utilizó el cuestionario de Ansiedad Estado-Rasgo. Resultados: Los resultados ponen de manifiesto la presencia de diferencias significativas entre las medidas de Ansiedad Estado en primera y segunda fase condicionadas por el sexo y el nivel académico; aunque los niveles de Ansiedad Estado y Ansiedad Rasgo no alcanzan en ningún momento puntuaciones de marcación negativa. Conclusiones: La experiencia vivida altera los niveles de Ansiedad Estado de de la muestra. Los niveles formativos superiores favorecen un afrontamiento positivo de la experiencia.
\end{abstract}

PALABRAS CLAVES: Ansiedad, emociones negativas, zona de operaciones, personal militar.

\begin{abstract}
Analysis of the emotional state of a Spanish army unit in an area of operations SUMMARY:

Introduction: Military people, due to the special caracteristics of their work and the places in which takes place, becomes a vulnerable group to suffer emotional changes, resultant of facing such situations. Materials and Methods: A total of 168 Members ( with an average age of 27 years, 12\% women and 88\% men) from the Spanish Army and Navy have been evaluated, displaced during 4 months in 2005 to the former Yugoslavia The objective of this research is the analysis of the changes generated in the negative emotional states of those individuals. Data collection took place in two different moments: the first one during the concentration pre-deployment period and the second one in a period just before the return to national territory. On both times we used the STAI anxiety test State-Trait Results: The result shows the presence of significant differences between the measures of state anxiety in the first and second phase influenced by the sex and academic level, although the levels of state anxiety and Trait anxiety do not reach at any time negative ratings. Conclusions: The lived experience alters levels of anxiety state of the sample. Higher level training enhance coping a positive experience.
\end{abstract}

KEYWORDS: Anxiety, negative emotions, operations area, military troop.

\section{INTRODUCCIÓN}

Bien como síntoma, manifestación o problemática en sí, el peso específico que la ansiedad ha ido adquiriendo en la dinámica vital de las personas se ha potenciado de una forma significativa.

Cualquier ser humano, cuando debe afrontar situaciones que cree superan sus propias capacidades, es altamente probable que acabe desarrollando una problemática (ya manifiesta, ya encubierta) que atente contra su salud mental, su rendimiento, relaciones personales y sociales, etc. ...

Si esta es una reacción factible en cualquier persona, cabría pensar que aquellos individuos que hacen de su labor cotidiana el abordaje de situaciones críticas se convierten en una población diana susceptible de padecer afecciones en la citada salud mental. Profe-

\footnotetext{
${ }^{1}$ Cap. Psicóloga. Gabinete de Psicología. Tercio de Armada San Fernando (Cádiz).

2 Departamento de Personalidad, Evaluación y Tratamiento Psicológicos. Facultad de Psico-
} logía. Universidad de Sevilla.

Dirección para correspondencia: Ana M $\mathrm{M}^{\mathrm{a}}$ Pérez Hidalgo. Población Militar San Carlos. 11100-San Fernando-Naval (Cádiz). 823-5305/ 956-599000 Ext 35305. Mail: aperhi1@ fn.mde.es

Recibido: 17 de noviembre de 2009

Aceptado: 25 de enero de 2011 sionales de las emergencias: bomberos, policías, personal sanitario, etc. ... así como otros que ocasionalmente se ven envueltos en estos operativos como es el caso del personal militar en misiones humanitarias, tanto a nivel nacional como internacional, acaban siendo los representantes más característicos.

Las tareas más usuales en estos grupos suelen ser diversas; aunque a nuestro criterio entendemos como englobables en 3 grupos: Apoyo (reconstrucción, reparto de alimentos, tareas policiales, coordinación y alojo de desplazados y regulación de tareas entre fuerzas militares y organizaciones humanitarias), Rescate (salvamento y evacuación de heridos de la zona cero, recuperación de cadáveres, reconstrucción e identificación de dichos cadáveres, etc....) y Abordaje (primeros auxilios en zona de impacto, atención médica en hospitales de campaña, apoyo psicológico, etc. ...). Diversos autores ${ }^{1,2}$ ya indican que ante este abanico de acciones no es extraño que cada una o varias de ellas puedan llegar a convertirse en una fuente de malestar para quien las ejecuta.

De otro lado, si las acciones son las enunciadas, cada una de ellas lleva implícito un riesgo bien Físico -tanto para sí mismos como para aquellos otros que integran el equipo- (resultado del desenvolvimiento en las «zonas de riesgo» o en las mismas «zonas calientes») bien Psíquico (impacto resultante de vivir-revivir y/o 
presenciar destrucción y dolor en otros o en propios compañeros, frustración e impotencia ante la evidencia de la incapacidad de evitar daños mayores, toma de decisiones que entrañan vida o muerte a terceros, tensión resultante de la propia urgencia de las intervenciones o de la autoexigencia de reducir al máximo los márgenes de error). A todo ello hay que adicionarle las acciones descoordinadas como resultado de la carencia de información acerca de lo que está ocurriendo en otros lugares y que generan inseguridad.

Las consecuencias de las Acciones y los Riesgos se encuentran a su vez condicionadas por Factores Profesionales (nivel de responsabilidad dentro de la organización o equipo, formación, experiencia en el desempeño del puesto y, dependiendo de lo anterior, área geográfica de intervención) y Personales (edad, sexo, características de personalidad, estrategias de afrontamiento prevalentes, nivel de desarrollo personal, apoyo social percibido y real, etc...).

El peso y las repercusiones de los estresores enunciados más arriba van a verse sometido al influjo de unas variables moduladoras ${ }^{3}$ que de forma resumida podemos distinguir entre: génesis y carácter del tipo de situación crítica, personas afectadas, características de las heridas sobre las que se interviene, disponibilidad de medios y grado de organización de las intervenciones. De la interacción, por tanto entre Tareas y Riesgos bajo el condicionamiento de los Factores Personales y Personales y con la incidencia de las Variables Moduladoras indicadas sería posible conjugar un amplio espectro de posibilidades.

Las situaciones de crisis que nos ocupan, se tratan de acontecimientos que si bien afectan de forma marcada a las víctimas directas, baste recordar en este sentido toda la bibliografía existente desde el siglo XIX hasta la actual DSM-IV TR (APA, 2005) ${ }^{4}$ o la esperada DSM V (APA, 2010) $)^{5}$ no es menos cierto que aquellas personas que también se ven implicadas (ya sea como personal de ayuda y apoyo ya como simples espectadores) acaban desarrollando una problemática paralela. Se trata del denominado Estrés Traumático Secundario $^{6,7}$; Agotamiento Emocional ${ }^{8}$ y Ruptura o Replanteamiento de Creencias ${ }^{9}$ vienen a completar un cuadro suficientemente conocido y en el que la ansiedad se convierte en un síntoma destacado.

Ha sido característico, hasta no hace demasiado tiempo, no centrar la atención en los profesionales que trabajan con víctimas de muy diverso tipo. El interés por conocer la problemática desarrollada en las citadas víctimas, sus reacciones y posteriores secuelas han focalizado el interés de la investigación. Se aceptaba la evidencia de que estaban sometidos a estrés y la consecuente insatisfacción resultante de sus intervenciones no siempre eficaces; pero los componentes emocionales quedaban en un segundo plano.

En un momento posterior la atención se ha orientado hacia los posibles estresores que rodean estas intervenciones profesionales. Se ha demostrado el gran peso de los estresores laborales en la salud mental del personal de emergencias ${ }^{10} \mathrm{y}$ su capacidad de predecir la satisfacción laboral ${ }^{11}$.

No obstante, el interés por los factores personales entendidos como variables moduladoras de las situaciones estresantes que el personal de emergencias debe afrontar también posee una cierta tradición iniciada en $1997^{12}$ y su interés por la empatía y el manejo de las competencias emocionales ${ }^{13}$ que en las últimas fechas ha adquirido una mayor importancia a la hora de llevar a cabo análisis pormenorizados. En este sentido destacan en nuestro país los recientes trabajos ${ }^{14,15}$, los cuales demuestran la importancia de diversas variables de personalidad como factores que modulan los efectos del estrés traumático secundario.
El personal militar que se desplaza no sólo a zona de operaciones bélicas, si no también a zonas de crisis en las que las tareas de ayuda, reconstrucción y apoyo a la población civil acaban teniendo que afrontar unos estresores que bien por su impacto puntual (acciones de combate o intervención en situaciones críticas) o por su prolongación a lo largo del tiempo (despliegues en zona de operaciones en periodos de 4 a 6 meses) acaban generando unas secuelas que guardan un paralelismo con lo descrito en el caso del personal civil de emergencias. La reducción en la eficacia que estos problemas de salud mental pueden generar e incluso el coste económico y social que dimana de ellos merecen cada vez más atención. En este último caso cabe reseñar un estudio $^{16}$ en 2009 sobre los gastos que conllevan de los Trastornos por Estrés Post-Traumático (TEPT) en combatientes.

Otro estudio realizado en el $2010^{17}$ contrasta los cambios en la personalidad de militares profesionales norteamericanos desplegados en Irak y llegan a la conclusión de que los niveles altos de ansiedad se mantienen meses después del alejamiento de la zona crítica.

Diversos autores ${ }^{18}$ llegan a similares resultados con combatientes en Irak y Afganistán. Línea argumental que también mantienen en otro estudio ${ }^{19}$ en el 2007 donde se percibieron diferentes secuelas entre militares dedicados a tareas de combate y los orientados a acciones pacificadoras.

En vista de las conclusiones de la bibliografía consultada, parece evidente que nadie vuelve ileso de las zonas cero de intervención (ya sea militar o civil). En nuestro estudio nos planteamos como objetivo no detectar la presencia de TEPT o de cualquier otra alteración de índole patológica, dadas las características de las acciones a desempeñar, pero si los posibles cambios emocionales derivados del afrontamiento de las situaciones especiales. En este sentido la ansiedad ha sido elegida como variable diana, considerando que como resultado de la estancia en zona de operaciones tendrá lugar un incremento de los niveles de ésta, recurriendo al sexo y la formación cultural como variables secundarias de análisis en cuanto que pueden modular los efectos.

\section{MATERIAL Y MÉTODOS}

\section{Muestra}

En el estudio han participado los 168 componentes de una unidad del ejército español, que integraron la SPFOR XV y que desde comienzos de Mayo a principios de Setiembre de 2005 permanecieron en la antigua exYugoslavia, teniendo su base en Mostar-Aeropuerto Bosnia-Herzogovina. Durante su periodo de estancia su misión consistió en facilitar ayuda humanitaria y evaluar el impacto de la guerra.

Se trata de un contingente en el que la procedencia geográfica de sus integrantes se encuentra muy repartida a lo largo del territorio nacional, sin que podamos determinar el predominio de una Comunidad Autónoma particular.

En cuanto a su distribución en base al sexo, hallamos que 148 $(88,09 \%)$ son varones y $20(11,90 \%)$ mujeres. Se trata de unos porcentajes muy similares a los existentes en el presente dentro de las Fuerzas Armadas españolas.

Desde el punto de vista de los niveles culturales, la tabla 1 recoge la forma en la que la muestra se reparte en base a este aspecto.

Las edades oscilan entre los 18 y los 52 años, siendo la edad media general de 27 y su desviación típica de 7,75. La organización de esta variable en base al sexo se puede consultar en la tabla 2. 
Tabla 1. Nivel cultural de la muestra.

\begin{tabular}{|lc|}
\hline Nivel de estudios & N (\%) \\
\hline Primarios & $99(58,93 \%)$ \\
Medios & $46(27,38 \%)$ \\
Universitarios & $23(13,7 \%)$ \\
\hline
\end{tabular}

Tabla 2. Edades de la muestra.

\begin{tabular}{|lccc|}
\hline & Hombres & Mujeres & Global \\
\hline Media & 27 & 24 & 27 \\
Desviación Típica & 8,15 & 3,7 & 7,75 \\
\hline
\end{tabular}

Finalmente, el GRÁFICO 1 informa acerca del peso específico de los distintos empleos militares en el contingente que nos ocupa. Tal y como se puede apreciar, la distribución piramidal, característica de la organización de origen, se mantiene de forma evidente.

\section{Instrumentos}

Para estudiar la ansiedad se empleo el Cuestionario de Ansiedad Estado-Rasgo (STAI A/E, A/R) de Spielberger, Gorsuch y Lushene (1970) adaptado a la población española ${ }^{20}$ en 1982. El cuestionario STAI lo constituyen 2 escalas independientes, constituyendo éstas dos formas de aproximación a la ansiedad: Estado (STAI/E) y Rasgo (STAI/R), cada una de ellas formada por 20 items con 4 opciones de respuesta, siendo su forma de administración de tipo autoaplicada.

\section{Procedimiento}

El estudio se ha caracterizado por dos fases diferenciadas en cuanto al momento de evaluación, constituyendo así una secuencia pre/post.

- Primera medida (Fase A). Se llevó a cabo el segundo día de estancia en el acuartelamiento considerado como Centro de Concentración. Se dispuso de un salón de actos en el que en un primer momento se informó acerca de los objetivos y las características de la investigación, así como de la voluntariedad en cuanto a la participación y de su derecho a ser informados individualmente de los resultados generales y de los propios de cada uno de ellos. Se les comunicó también la posibilidad de poder recurrir a poner claves o seudónimos con el fin de mantener el anonimato, caso de desearlo. La evaluación en sí tuvo lugar después de haber dejado un espacio temporal de 15 minutos de libre movilidad por el salón y sus alrededores a fin de que aquellas personas que no quisieran colaborar no se sintieran presionados en ningún momento.

- Segunda medida (Fase B). Se procedió a ella, ya en zona de operaciones, 7 días antes del regreso a España. Se volvieron a recordar las características del estudio y se insistió en que aquellos que habían empleado seudónimos y/o claves en la primera ocasión volvieran a usar las mismas. También se trató de una aplicación colectiva en el comedor de la base y empleando la misma dinámica ya descrita.

\section{RESULTADOS}

Para el análisis estadístico de los datos se ha hecho uso del programa estadístico informatizado SPSS 14.0. Se estimaron los esta- dísticos descriptivos básicos (medias, medidas de tendencia central, dispersión y forma), así como una comparación de medias en grupos independientes ( $\mathrm{T}$ de Student Fisher) y finalmente un Análisis de Varianza unifactorial ínter grupos.

Los valores promedio, tanto en la muestra global como distribuidos en función del sexo y de la fase de estudio, pueden consultarse en la tabla 3.

Tabla 3. Valores promedio en la escala A/E y A/R del STAI, en ambas fases del diseño.

\begin{tabular}{llllllc|}
\hline & \multicolumn{2}{c}{ Global } & \multicolumn{2}{c}{ Hombres } & \multicolumn{2}{c|}{ Mujeres } \\
\cline { 2 - 7 } & Media & $\sigma$ & Media & $\sigma$ & Media & $\sigma$ \\
\hline Estado 1 & 20,12 & 17,94 & 20,66 & 18,04 & 16,15 & 17,11 \\
Estado 2 & 28,55 & 26,19 & 29,22 & 25,96 & 23,65 & 27,98 \\
Rasgo 1 & 18,53 & 19,85 & 18,93 & 19,73 & 15,60 & 21,01 \\
Rasgo 2 & 19,35 & 24,48 & 19,52 & 24,73 & 18,10 & 23,06 \\
\hline o= desviación típica.
\end{tabular}

Un primer análisis nos permite poner en evidencia que, empleando los valores promedio de la muestra de baremación española en el caso de los varones (20,54 en A/E y 20.19 en $A / R)$ y en el de las mujeres (23,30 para A/E y 24,99 en A/R), tanto unos como otros se sitúan por debajo de estos niveles -tanto en $\mathrm{A} / \mathrm{E}$ como en $\mathrm{A} / \mathrm{R}$ - en las primeras aplicaciones, mientras que en las segundas, la submuestra masculina asciende de forma marcada en $\mathrm{A} / \mathrm{E}$ existiendo un cierto incremento; aunque no tan marcado en A/R. Por su parte, los componentes femeninos del contingente aunque si muestran incrementos tanto en $\mathrm{A} / \mathrm{E}$ como en $\mathrm{A} / \mathrm{R}$ en territorio nacional y en zona de operaciones, siempre se encuentran dentro de los niveles medios de las muestras de referencia. En ambos grupos es destacable la presencia de unas desviaciones típicas elevadas, mucho más en el caso de las mujeres.

La tabla 4 facilita la distribución de puntuaciones medias en función de los niveles culturales y las secuencias de aplicación. Se evidencia como en las personas con formación primaria y media hay un incremento por encima de los valores normativos en los niveles de $\mathrm{A} / \mathrm{E}$, mientras que en los universitarios no sólo no se produce tal aumento, sino una disminución.

Tabla 4. Valores promedio en la escala A/E y A/R del STAI, según los niveles culturales en ambas fases del diseño.

\begin{tabular}{|lcccccc|}
\hline & \multicolumn{2}{c}{ Primarios } & \multicolumn{2}{c}{ Medios } & \multicolumn{2}{c|}{ Universitarios } \\
\cline { 2 - 7 } & Media & $\sigma$ & Media & $\sigma$ & Media & $\sigma$ \\
\hline Estado 1 & 22,55 & 19,00 & 16,07 & 15,29 & 17,78 & 17,04 \\
Estado 2 & 31,95 & 27,20 & 29,41 & 27,26 & 12,22 & 7,53 \\
Rasgo 1 & 21,13 & 20,69 & 15,02 & 19,38 & 14,35 & 15,57 \\
Rasgo 2 & 20,55 & 24,39 & 21,00 & 28,21 & 10,91 & 13,56 \\
\hline
\end{tabular}

$\sigma=$ desviación típica.

En la tabla 5 es posible verificar como, con un nivel de significación .05 existen diferencias en $\mathrm{A} / \mathrm{E}$ cuando se contrastan las medias globales de la muestra en las 2 fases establecidas, cosa que no ocurre en el caso de $\mathrm{A} / \mathrm{R}$, cuestión que resultaba esperable de antemano.

En cuanto la influencia de la variable sexo, en el caso de A/E y $\mathrm{A} / \mathrm{R}$ tanto en la primera como en la segunda fase no se han detec- 
Tabla 5. Contraste de los valores medios alcanzados en $A / E$ en los dos momentos de evaluación.

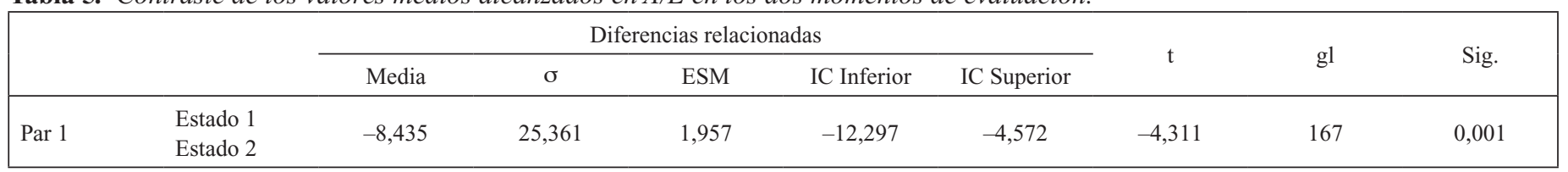

$\sigma=$ desviación típica. ESM = error estándar de la media. $\mathrm{IC}=$ Intervalo de confianza al 95\%. $\mathrm{t}=$ Distribución t de Student. gl = grados de libertad. Sig.= Significación bilateral.

Tabla 6. Comparación de valores medios entre A/E 1-A/E 2 y A/R 1-A/R 2, en varones, en los dos momentos del estudio.

\begin{tabular}{|c|c|c|c|c|c|c|c|c|c|}
\hline & & \multicolumn{5}{|c|}{ Diferencias relacionadas } & \multirow{2}{*}{$\mathrm{t}$} & \multirow{2}{*}{$\mathrm{gl}$} & \multirow{2}{*}{ Sig. } \\
\hline & & Media & $\sigma$ & ESM & IC Inferior & IC Superior & & & \\
\hline Par 1 & $\begin{array}{l}\text { Estado } 1 \\
\text { Estado } 2\end{array}$ & $-8,561$ & 25,504 & 2,096 & $-12,704$ & $-4,418$ & $-4,084$ & 147 & 0,001 \\
\hline Par 2 & $\begin{array}{l}\text { Estado } 1 \\
\text { Estado } 2\end{array}$ & $-0,595$ & 25,616 & 2,106 & $-4,756$ & 3,567 & $-0,282$ & 147 & 0,778 \\
\hline
\end{tabular}

$\sigma=$ desviación típica. $\mathrm{ESM}=$ error estándar de la media. $\mathrm{IC}=$ Intervalo de confianza al 95\%. $\mathrm{t}=$ Distribución $\mathrm{t}$ de $\mathrm{Student} . \mathrm{gl}=$ grados de libertad. Sig.= Significación bilateral.

tado diferencias significativas entre hombres y mujeres mediante el uso del estadístico «t» de Student Fisher de comparación de medias independientes.

La tabla 6 recoge como se detecta la presencia de un incremento significativo (a nivel .05) entre A/E1 y A/E2 en los varones, mientras que en el caso de A/R los cambios no son apreciables. En el caso de la submuestra femenina las posibles diferencias no llegan a alcanzar valores que merezcan la consideración de importantes en ninguna de las medidas de la ansiedad.

Las tablas 7 y 8 dejan de manifiesto que en niveles culturales primarios (tabla 7) y medios (tabla 8) únicamente es posible detectar una incrementación en las puntuaciones logradas en $\mathrm{A} / \mathrm{E}$, mientras que las medidas de A/R se mantienen niveladas. En el caso de los individuos con niveles culturales superiores (universitarios) sucede lo contrario a la tendencia ya indicada, es decir, la ansiedad estado y rasgo decrece (aunque estadísticamente este decrecimiento no resulta revelador).

Finalmente, la tabla 9 reproduce los valores del ANOVA efectuado con el fin de verificar de forma efectiva el papel que los citados niveles culturales juegan en las disimilitudes evidentes entre $\mathrm{A} / \mathrm{R}$ y $\mathrm{A} / \mathrm{E}$

\section{DISCUSIÓN}

Los militares que conforman la muestra que se ha estudiado acudieron a Bosnia-Herzegovina en el año 2005, lo cual supone, desde el punto de vista de seguridad, que su presencia no estaba asociada a ciertas acciones de combate, por cuanto los acuerdos de Dayton tuvieron lugar en 1995. Tareas policiales, de control, apoyo y reconstrucción constituyeron sus principales actividades, si bien es cierto que tenían que desenvolverse en un entorno en el que la destrucción y sus secuelas (a niveles personales y físicos) todavía eran visibles en las poblaciones.

Los riesgos personales del contingente (a parte del que conllevan en sí las labores de control o policiales) estaban más vinculados a accidentes bien laborales bien viales, en este último caso referidos a los difíciles accesos a algunas de las localizaciones.

Esta descripción debe completarse con la presencia de otros posibles estresores derivados de los ritmos de trabajo, observación de niveles de tensión en compañeros como consecuencia de la convivencia estrecha durante un periodo amplio de tiempo, carencia del apoyo social conocido (familia, amigos, etc...), elaboraciones de carácter moral y ético como consecuencia de las situaciones experi-

Tabla 7. Comparación de valores medios entre A/E 1-A/E2 y A/R 1-A/R 2 en sujetos con estudios primarios.

\begin{tabular}{|c|c|c|c|c|c|c|c|c|c|}
\hline & & \multicolumn{5}{|c|}{ Diferencias relacionadas } & \multirow{2}{*}{$\mathrm{t}$} & \multirow{2}{*}{$\mathrm{gl}$} & \multirow{2}{*}{ Sig. } \\
\hline & & Media & $\sigma$ & ESM & IC Inferior & IC Superiort & & & \\
\hline Par 1 & $\begin{array}{l}\text { Estado } 1 \\
\text { Estado } 2\end{array}$ & $-9,404$ & 26,024 & 2,615 & $-14,594$ & $-4,214$ & $-3,596$ & 98 & 0,001 \\
\hline Par 2 & $\begin{array}{l}\text { Rasgo } 1 \\
\text { Rasgo } 2\end{array}$ & 0,586 & 27,913 & 2,805 & $-4,981$ & 6,153 & 0,209 & 98 & 0,835 \\
\hline
\end{tabular}

$\sigma=$ desviación típica. $\mathrm{ESM}=$ error estándar de la media. $\mathrm{IC}=$ Intervalo de confianza al 95\%. $\mathrm{t}=$ Distribución $\mathrm{t}$ de Student. gl = grados de libertad. Sig. $=$ Significación bilateral .

Tabla 8. Confrontación de valores medios entre A/E 1-A/E2 y A/R 1-A/R 2 en sujetos con estudios medios.

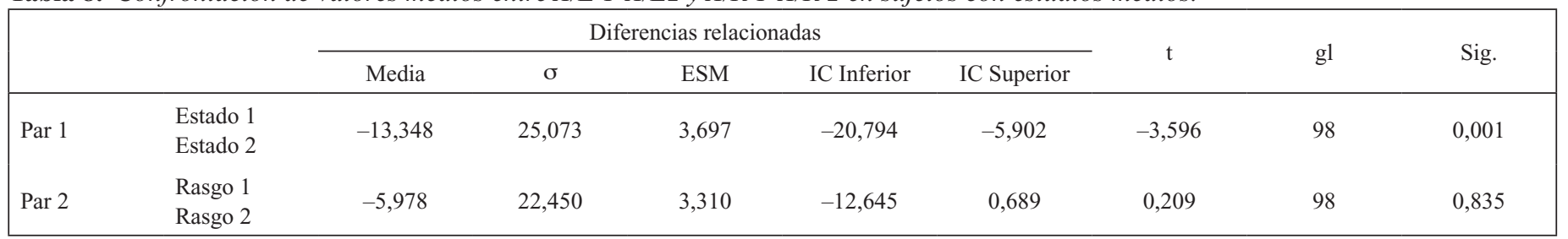

$\sigma=$ desviación típica. $\mathrm{ESM}=$ error estándar de la media. $\mathrm{IC}=$ Intervalo de confianza al 95\%. $\mathrm{t}=$ Distribución $\mathrm{t}$ de Student. $\mathrm{gl}=$ grados de libertad. Sig. $=$ Significación bilateral. 
Tabla 9. Análisis de varianza (ANOVA) unifactorial intergrupos.

\begin{tabular}{|c|c|c|c|c|c|c|}
\hline & & Suma de cuadrados & gl & Media cuadrática & $\mathrm{F}$ & Sig. \\
\hline \multirow[t]{3}{*}{ Estado 1} & Inter-grupos & 1464,356 & 2 & 732,178 & 2,308 & 0,103 \\
\hline & Intra-grupos & 52339,263 & 165 & 317,208 & & \\
\hline & Total & 53803,619 & 167 & & & \\
\hline \multirow[t]{3}{*}{ Rasgo 1} & Inter-grupos & 1638,363 & 2 & 819,181 & 2,105 & 0,125 \\
\hline & Intra-grupos & 64217,489 & 165 & 389,197 & & \\
\hline & Total & 65855,851 & 167 & & & \\
\hline \multirow[t]{3}{*}{ Estado 2} & Inter-grupos & 7313,705 & 2 & 3656,853 & 5,627 & 0,004 \\
\hline & Intra-grupos & 107233,813 & 165 & 649,902 & & \\
\hline & Total & 114547,518 & 167 & & & \\
\hline \multirow[t]{3}{*}{ Rasgo 2} & Inter-grupos & 1903,908 & 2 & 951,954 & 1,600 & 0,205 \\
\hline & Intra-grupos & 98186,372 & 165 & 595,069 & & \\
\hline & Total & 100090,280 & 167 & & & \\
\hline
\end{tabular}

$\mathrm{F}=$ Distribución $\mathrm{F}$ de Snedecor. gl = grados de libertad. Sig. = Significación bilateral.

mentadas, posible resurgimiento de secuelas resultado de situaciones traumáticas personales no superadas, etc...

El análisis de los datos pone de manifiesto diversas cuestiones iniciales.

La primera que destaca es la existencia de tan sólo un 11,90\% de mujeres. Es preciso, tener en cuenta, a modo de justificación de este valor, que la presencia de mujeres en las Fuerzas Armadas rondaba el 13\% en 2008 y que en 2006 dicho porcentaje en EUFOR BosniaHerzegovina era del $4,5 \%{ }^{21}$. Es decir, se trata de una realidad que en el caso de la muestra estudiada y del tiempo en el que ocurrió, aparece mejorada en relación a la situación que existía.

De otro lado y desde un punto de vista general, los niveles promedio de ansiedad, ya sea A/R ya A/E, a pesar de haber sufrido algunos incrementos estadísticamente significativos, no alcanzan unos valores que en función de los baremos españoles del cuestionario STAI se consideren propios de unos percentiles elevados.

Las puntuaciones alcanzadas en la variable Ansiedad Rasgo se ajustan a lo indicado, de hecho ninguna de las personas evaluadas mostró en cualquiera de las dos fases de evaluación la presencia de indicadores que hiciesen pensar en unos radicales basales destacables y que permitiesen predecir reacciones emocionales anómalas. En cuanto a la estabilidad manifestada por las medidas, se atiene a lo ya planteado por diversos autores ${ }^{20,22}$.

Esta circunstancia también debe hacernos pensar en la eficacia de los procesos selectivos previos a misiones que se llevan a cabo en cualquiera de los niveles de las Fuerzas Armadas.

Un estudio ${ }^{23}$ donde se comparan a militares noruegos y voluntarios civiles que entre 1992 y 1995 estuvieron desplegados en Yugoslavia en pleno conflicto bélico. Aunque las condiciones en nuestro estudio y en este otro citado no son similares, cabe destacar como en el caso de los civiles (por tanto no combatientes y con desarrollo de acciones propias de ayuda) se evidenciaban síntomas de ansiedad, depresión y fobias concretas; aunque sin llegar al nivel de TEPT (el cual si se manifestaba en los combatientes). Sin lugar a dudas, las distintas situaciones (las cuales conllevan niveles e intensidades diferentes) a afrontar tienen como consecuencia reacciones bien diferenciadas. En esta línea es posible recurrir al excelente trabajo ${ }^{24}$ con personal de emergencias (mayoritariamente no relacionado con el área de la seguridad) implicado directamente en los atentados del $11 \mathrm{M}$ en Madrid. Las reacciones peri traumáticas adversas (y la ansiedad se localiza entre ellas), tal y como estos autores las califican, son esperables en estos grupos profesionales, independientemente del hecho de poseer un adecuado entrenamiento, a causa del contacto continuado como resultado de la implicación en los acontecimientos.

Ya hemos insistido acerca de lo elevado de las desviaciones típicas en todos los análisis que se han realizado. Esa elevación hace pensar en una amplia diversidad de puntuaciones y que a todas luces obedecen a la influencia de diversas variables que aunque no se han contemplado dado lo reducido del grupo de estudio, no por ello son menos importantes. Características de personalidad, tipo de situaciones a afrontar (no todos los miembros de la muestra estuvieron sometidos a las mismas situaciones estimulares), evaluación subjetiva de las experiencias vividas y la confluencia de experiencia y formación constituyen el núcleo central al que señalamos.

Numerosos estudios ${ }^{14,25-28}$ inciden en el alto valor defensivo que poseen las personalidades resistentes, minimizando los efectos y modulando la gravedad de los síntomas que puedan llegar a experimentar los profesionales. En este mismo discurso, si esta modalidad de personalidad se ve apoyada por una evaluación subjetiva de los hechos traumáticos identificándolos como un reto a las propias capacidades, los efectos negativos esperados se acaban reduciendo ${ }^{29}$. Por el contrario, rasgos como el neuroticismo incrementan la vulnerabilidad $^{30,31 .}$

$\mathrm{Y}$ a se ha puesto de manifiesto en numerosas ocasiones ${ }^{32,33}$ que en la medida en la que los acontecimientos traumáticos a afrontar tienen un origen humano intencionado, el impacto y las subsecuentes reacciones en quienes lo experimentan de forma directa o indirecta es mayor.

En cuanto a la formación y experiencia, aunque nos ocuparemos de ellas más adelante, hay que reconocer que se trata de premisas básicas e imprescindibles; aún cuando no exclusivas, para evitar implicaciones emocionales con las victimas ${ }^{34}$.

Las tablas 3, 5 y 6 permiten por tanto evidenciar, siguiendo los planteamiento del manual utilizado ${ }^{22}$, que los componentes de nuestro estudio se muestran sensibles a situaciones experienciales marcadas, ya por la tensión ya por el estrés, siendo la A/E una consecuencia de ello. No hay que olvidar que se trata de un contingente que ha estado desplegado en zona de operaciones durante varios meses, facilitándose así posibles reexperimentaciones. De forma más concreta es en los varones donde se aprecia esto de manera más característica. Todo ello sin olvidar que dichos valores tampoco llegan a unas alturas que hagan temer consecuencias importantes.

En la submuestra de mujeres, a pesar de detectarse un aumento en las puntuaciones medias en $\mathrm{A} / \mathrm{E}$, éste no adquiere significación estadís- 


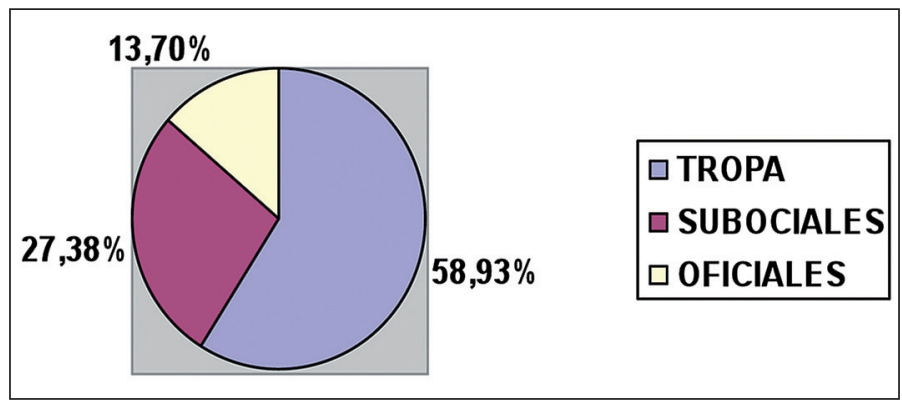

Figura 1. Organización del contingente en base a los empleos.

tica. ¿Se trata de un grupo más resistente? Hay un hecho esclarecedor si consultamos de nuevo las desviaciones típicas. Tanto en la muestra considerada de forma global como secuenciada en base al sexo, dichas desviaciones son muy importantes, especialmente en las 20 mujeres. Dicho de otra forma, la variabilidad de las respuestas es tan destacada que llega a superar los valores medios, condicionando las conclusiones que pudieran llegar a hacerse en este ámbito concreto.

Plantear posibles diferencias, en cuanto a niveles de afectación condicionadas por el sexo sería extremadamente arriesgado a partir de los resultados de la presente investigación, dado que la prevalencia de uno u otro sexo viene condicionada por las características de la organización en la que se encuentran integrados los componentes y por la propia idiosincrasia de ésta.

La formación cultural es otra de las variables alrededor en la que se gestan discrepancias reseñables.

La tabla 1 recoge el predominio de los componentes con estudios primarios $(58,93)$ frente a los medios $(27,38)$ y universitarios $(13,7)$, todo ello resulta esclarecedor si recurrimos la figura 1 y comprobamos que dichas diferencias formativas coinciden a su vez con la distribución de empleos y esta última es acorde a lo habitual y establecido en una organización de carácter militar.

Asimismo, las tablas 7, 8 y 9 ponen en evidencia que personal de tropa y suboficiales (con equivalencias a formación primaria y media) son más proclives a reacciones emocionales negativas de carácter transitorio como consecuencia a tener que arrostrar situaciones especiales de forma continuada. ¿La posesión de un nivel cultural ayuda a un mejor procesamiento de la citas situaciones o todo obedece a que esta última submuestra posee un $\mathrm{N}=23$ y ello condiciona los valores?

Un estudio realizado en $1998^{35}$ afirma que, entre otras variables que pueden ayudar a predecir la aparición de estrés post-traumático en personal de emergencias, es posible destacar el bajo nivel educativo, un estudio ${ }^{24}$ realizado tras el $11 \mathrm{M}$ destaca como de la muestra de este mismo personal que estudian tras los atentados, son aquellos que disponen de un menor nivel cultural (en esta ocasión policías) los que se muestran más sensibles a desarrollar secuelas posteriores frente a lo que ocurre en personal sanitario, de salud mental y social.

Otro aspecto a destacar, es que las desviaciones típicas, como en las ocasiones anteriores, muestran unos niveles excesivamente elevados en las 3 submuestras.

Sexo y nivel cultural aparecen como elementos vinculados a posibles reacciones de sensibilización ante situaciones generadoras de tensión emocional vivenciadas de forma continuada. No obstante dichas reacciones no provocaron en su momento la necesidad de intervención del personal sanitario ni tampoco han alcanzado en las medidas objetivas que nos hagan pensar en valores extremos.
Son varias las posibles variables que podrían estar incidiendo en el caso especial de la muestra que nos ocupa y que abrirán el camino a próximas investigaciones.

Las dimensiones o rasgos básicos de personalidad (que condicionan las interpretaciones de los hechos críticos) se constituyen en el núcleo central alrededor del cual giran:

- Organización de pertenencia en la que la propia cultura organizacional tiene un peso específico tan marcado que sirve como elemento de contención. La regulación de acciones, la distribución de responsabilidades, recursos, principios, estilo relacional, etc... se encuentran estrictamente delimitados y regulados.

- Formación. El establecimiento de una fase de concentración previa al despliegue en la que la preparación y la información acerca de las acciones a poner en marcha, coordinación de equipos y establecimiento de relaciones de cohesión y alianzas van a disminuir la aparición de la evidente «ansiedad anticipatoria»; pero a la vez va a ayudar a prevenir posteriores reacciones negativas. En esta dirección redundan diversos trabajos ${ }^{36,37}$.

\section{CONCLUSIONES}

Las principales conclusiones obtenidas en esta investigación:

- Los niveles de Ansiedad Estado de la muestra, han sufrido un incremento entre la primera y la segunda evaluación efectuada, la experiencia vivida afecta a los miembros del contingente.

- Los valores de Ansiedad Rasgo de la muestra, no han sufrido una variación significativa entre ambas evaluaciones.

- No se pueden establecer conclusiones teniendo en cuenta la variable sexo, debido a la escasa representatividad femenina en la muestra.

- El nivel académico superior favorece un mayor control de la ansiedad.

\section{BIBLIOGRAFÍA}

1. Ursano, R.J.; Fullerton, C.S. y Norwood, A.E. (Eds) (2003). Terrorism and disaster. Individual and community mental health interventions. Cambridge: Cambridge University Press.

2. Young, B.H.; Ford, J.D.; Ruzek, J.I.; Friedman, M.J. y Gusman, F.D. (1998). Disaster mental health services: A guidebook for clinicians and administrators. Palo Alto, CA: Departament of Veterans Affairs. National Center for PTSD

3. Rodríguez González, J. M. y Pérez Hidalgo, A. (2008). Abordaje de víctimas de emergencias. Reflexiones previas a la intervención de los sujetos activos. En Universidad SEK y Academia de Artillería (Eds.). Actas del III Congreso Nacional sobre Seguridad, Información y Defensa: Comunicación, conflictos y políticas de Seguridad y Defensa. Segovia: Biblioteca de Ciencia y Artillería, 61-72.

4. American Psychological Association (2005). Manual diagnóstico y estadístico de los trastornos mentales: texto revisado. Barcelona: Masson.

5. American Psychological Association (2010). Diagnostic and statistical manual of mental disorders. DSM 5. http://www.dsm5/Pages/Default.aspx.

6. Figley, C.R. (1995). Compassion Fatigue: Coping with secundary traumatic stress disorder in those who treat the traumatized. Nueva York: Brunner/Mazel Publishers

7. Stamm, B. H. (1995). Secondary traumatic stress: Self-care sigues for clinicians, researchers and educador. Baltimore: The Sidran Press

8. Stamm, B.H. (2002). Measuring compassion satisfaction as well as fatigue: Developmental history of the compassion satisfaction and fatigue test. En Figley, C.R.: Treating compassion fatigue. Psychosocial stress series, 24, 107-109. Nueva York: Brunner-Routledge.

9. Jaoff-Bulman, R. (1992). Shattered assumptions. New York: free Press. 


\section{Análisis del estado emocional de una unidad del ejército español en zona de operaciones}

10. Kop, N., Euwema, M., y Schaufeli, W. (1999). Burnout, job stress and violent behaviour among Dutch police officers. Work and Stress, 13, 326-340.

11. Brough, P. (2004). Comparing the influence of traumatic and prganizational stressors on the psychological heath of police, fire and ambulance officers. International Journal of Stress Management, 11 (3), 237-244.

12. Figley, C.R. (1997). Burnout in Families: The systemic costs of caring. Nueva York: CRC Press.

13. Moran, C.C. (1999). Differential influences of coping humour and humour bias on mood. Behavioural Medicine, 25, 36-42.

14. Moreno-Jiménez, B., Morett, N., Rodríguez-Muñoz, A. y Morante, M. E. (2006) La personalidad resistente como variables moduladora del síndrome de burnout en una muestra de bomberos. Psicothema, 18 (3), 413-418.

15. Moreno-Jiménez, B., Morante, M. E., Rodríguez-Carvajal, R. Y Rodríguez Muñoz, A. (2008). Resistencia y vulnerabilidad ante el trauma. El efecto moderador de las variables de personalidad. Psicothema, 20 (1), 124-130.

16. Tanielian, T. (2009). Assessing combat exposure and post-traumatc estress disorde in troops and stimating the cost o society. Santa Mónica: RAND C ${ }^{\circ}$.

17. Buls, A. y Vorobjovs, a. (2010). Transformation of the soldiers personality in the internacional operation theatre and the postoperational stage. ATI-Applied Technologies \& Innovatios, 1 (1), 33-42

18. Hoge, Ch. W., Castro, C.A., Messer, S.C., McGurk, D., Cotting,D.I y Koffman, R.L. (2004). Combat duty in Iraq and Afganistán, mental health problems, and barriers to care. New Englan Journal od Medicina, 351, (1), 13-22

19. Sareen, J. Cox, B.J., Afifi, T.O., Stein, M.B., Belik, S-L., Meadows, G. y Asmundson, G.J.G. (2007). Combat and peacekeeping operation in relation to prevalence of mental disordes and perceived need for mental health care. Archives of General Psychiatry, 64 (7), 843-852.

20. Seisdedos, N. (1982). STAI. Manual del cuestionario de ansiedad Estado-Rasgo. TEA: Madrid.

21. Val, C. Del. (2009). La mujer militar en las misiones de paz de la Unión Europea y las Naciones Unidas. En Gómez, M. y Sepúlveda, I. (Eds.): Las mujeres militares en España (1988-2008) (pp. 201-218). Madrid: Instituto Universitario General Gutiérrez Mellado-UNED.

22. Spielberger, C.D., Gorsuch, R.L. y Lushene, R.(1970). STAI. Manual for the satae-trait anxiety inventory. Palo Alto. CA: Consulting Psychologists Press.

23. Kaspersen, M. y Matthiensen, S. (2002). Síntomas de estrés postraumático entre los soldados de Naciones Unidas y el personal perteneciente al voluntariado. European Journal of Psychiatry, 17 (2), 69-77.

24. González, H., Miguel-Tobal, A., Cano, A. e Iruarrizaga, I. (2004). Efectos de la exposición a eventos traumáticos en personal de emergencias: consecuencias psi- copatológicas tras el atentado terrorista del 11-M en Madrid. Ansiedad y Estrés, $10(2-3), 207-217$.

25. Funk, S.C. (1992). Hardiness: A review of theory and research. Health Psychology, 11 (5), 335-345

26. Hills, H. y Norvell, N. (1991). An examination of hardiness and neuroticism as potencial moderators of strees outcomes. Behavioral Medicine, 17 (1), 31-38.

27. Kobasa, S.C. (1982). The hardy personality: toward a social psychology of stress and health. En G.S. Sanders y J. Suls (Comp.): Social psychologyof health and illness. Hillsdale, N.J.: Lawrence Erlbaum.

28. Moreno-Jiménez, B., González, J. L., Garrosa, E. y Peñacoba, C. (2001). Variables sociodemográficas en el proceso de desgaste profesional de enfermería. Rol de Enfermería, 25 (11), 18-26.

29. Solkova, I. y Tomakek, P. (1994). Daily stress doping strategies an effect of hairdness. Studio Psychologica, 36, 390-392.

30. Bramse, I., Dirkzwager, A.J.E. y van der Ploeg, H.M. (2000) Predeployment personality traits and exposure to trauma as predictors af postraumatic strss symptons: A perspective study of former peacekeepers. American Journal of Psychiatry, 157, 111-1119.

31. Schnurr, P.P., Vielhauer, M.J. (1999). Personality as a risk factor for PTSD. En R. Yehuda (Ed.), Risk factors for postraumatic stress disorder (pp. 191-222). Washington D.C: American Psychiatric Press.

32. Norris, F.H., Friedman, M.J., Watson, P.J., Byrne, C.M., Díaz, E y Kaniasty, K. (2002). 60000 disaster victims speak: Part I. An empirical review of the empirical literature. 1981-2001. Psychiatry, 65, 207-239.

33. Norris, F.H., Friedman, M.J. y Watson, P.J. (2002). 60000 disaster victims speak: Part II. Summary and implications of the disaster mental health research. Psychiatry, 65, 240-260

34. Jonson, A. y Segesten, K. (2003). The meaning of traumatic events as described by nurses in ambulante service. Accident and Emergency Nursing, 11, 141-152.

35. Epstein, R.S., Fullerton, C.S. y Ursano, R.J. (1988). Posttraumatic stress disorder following ana ir disaster: a prospective study. American Journal of Psychiatry, $155,934-938$.

36. Merino Soto, C., Manrique Borjas, G., Angulo Ramos, M., e Isla Chavez, N (2007). Indicador de estrategias de afrontamiento al estrés: análisis normativo y de su estructura factorial. Ansiedad y Estrés, 13 (1), 24-39.

37. Moya-Albiol, L. y Occhi, S. (2007). Sintomatología del Trastorno por Estrés Postraumático y empatía en población no directamente afectada tras los atentados terroristas de Madrid del 11 de Marzo de 2004. Ansiedad y Estrés, 13 (2-3), 269-281. 